\title{
Molecular investigation of bacterial communities on intravascular catheters: not just Staphylococcus anymore
}

\author{
Li Zhang $^{1 *}$, John Gowardman ${ }^{1,2}$, Mark Morrison ${ }^{3}$, Lutz Krause ${ }^{4}$, Elliott G. Playford ${ }^{5}$; Claire \\ M. Rickard ${ }^{1,5}$ \\ ${ }^{1}$ Centre Health Practice Innovation, Griffith University, Brisbane, Australia; \\ ${ }^{2}$ Department of Intensive Care Medicine, Royal Brisbane Women's Hospital, Brisbane,
} Australia;

${ }^{3}$ University of Queensland Diamantina Institute, Brisbane, Australia;

${ }^{4}$ Bioinformatics Laboratory, Queensland Institute of Medical Research, Brisbane, Australia;

${ }^{5}$ Infection Management Services, Princess Alexandra Hospital, Brisbane, Australia

${ }^{*}$ Correspondence and requests for reprints should be addressed to Dr. Li Zhang, N48 Nathan Campus, Griffith University, 170 Kessels Road, Nathan QLD 4111, Australia. Tel: +61 7 37357272; Fax: +61 73735 3560; E-mail:li.zhang@griffith.edu.au.

Running title: Bacterial communities on intravascular catheters. 


\begin{abstract}
Intravascular catheter-related bloodstream infections (IVC-BSIs) are associated with significant morbidity and mortality. Culture-independent molecular approaches can reveal and capture the composition of complex microbial communities, and are now being used to reveal "new" pathogens as well as the polymicrobial nature of some infections. Patients with concurrently sited arterial and central venous catheters whom had clinically suspected IVCBSIs, were examined by high-through put sequencing of microbial 16S rRNA. An average of 100 operational taxonomic units (OTUs, phylotypes) was observed on each IVC, indicating that IVCs were colonized by complex and diverse bacterial communities. Ralstonia $(53 \%$ of 16S rRNA sequences), Escherichia group (16\%), Propionibacterium (5\%), Staphylococcus (5\%), and Streptococcus (2\%) were the most abundant genera. There was no statistically significant difference in the bacterial communities examined from arterial and central venous catheters; from those with and without systemic antibiotic treatment; or from conventionally colonised and uncolonised IVCs. The genome of the predominant bacteria, R. pickettii AU1208, was found to encode resistance to antimicrobial drugs of different classes. In addition, many encoded gene products are involved in quorum sensing and biofilm formation that would further contribute to increased antimicrobial drug resistance. Our results highlight the complex diversity of microbial ecosystems on vascular devices. High-throughput sequencing of 16S rRNA offers an insight into the pathogenesis of IVC-related infections, and opens up scope to improve diagnosis and patient management.
\end{abstract}

Keywords: intravascular catheter; catheter related bloodstream infections; biofilm; antibiotic resistance; high-throughput sequencing. 


\section{Introduction}

2 Intravascular catheters (IVCs), including central venous catheters (CVCs) and arterial

3 catheters (ACs), are the most frequently used invasive medical devices in hospitals [1].

4 However, IVCs are associated with life threatening bloodstream infections (IVC-BSIs) which

5 have high associated rates of morbidity, mortality and additional personal and medical costs

$6 \quad[2,3]$.

7 Bacteria cause IVC-related infections when they gain access to the surface of IVCs and

8 establish biofilm, allowing sustained surface colonization and ultimately, dissemination into

9 the bloodstream leading to IVC-BSI. Early detection and adequate treatment of causative

10 pathogens is critical for a favourable outcome, yet the majority of patients with a suspected

11 catheter-related infection yield negative diagnostic investigations, necessitating empiric,

12 rather than optimal, antimicrobial therapy [4].

13 Unfortunately, less than $1 \%$ of bacteria in nature can be recovered using culture-based

14 methods [5]. Our previous studies have shown that many fastidious bacteria on medical

15 devices, potentially responsible for sepsis, are not diagnosed using current standard culture

16 examination as used in hospital laboratories [6]. Thus the most frequently isolated bacteria

17 might not be the dominant bacteria on colonised IVCs, or, indeed be responsible for many

18 patients' clinical conditions. The next generation sequencing now provides a key approach by

19 which to examine these complex microbial communities in a high-throughput manner.

20 Importantly, these approaches can reveal, and capture, the genetic potential present in

21 complex microbial communities without having to isolate and culture the microorganisms.

22 The cultivation-independent molecular approaches are now being used to reveal "new"

23 pathogens, as well as the polymicrobial nature of some infections $[7,8]$.

24 Current strategies for control of IVC-BSI have focused more heavily on CVCs than ACs [9-

25 11]. However, the rate of IVC-BSIs arising from ACs has recently been demonstrated to be 
1 comparable to that of CVCs [12-14], thereby rendering ACs an important device to study.

2 Little is known as regards to the microbial colonisation of CVCs and ACs sited concurrently

3 in the same patient as is a common situation in the intensive care unit (ICU) patient where

4 most patients need both IVCs. Despite the differing circulations and use of the two device

5 types, it is possible that bacteria which colonize one device may also colonize other

6 concurrently sited IVCs, in similar fashion, since the skin is the common portal of entry.

7 Because CVCs have been considered a higher infective risk than ACs, for patients with

8 suspected IVC-BSI, clinicians may remove the CVC but not the AC. Even if both catheters

9 are removed, it may be that the $\mathrm{CVC}$ is only sent for investigation, despite the $\mathrm{AC}$

10 contributing equally to the infection, and being important for diagnosis and treatment. The

11 main aim of this study was to test this hypothesis and evaluate the bacterial profiles on the

12 surfaces of both ACs and CVCs removed concurrently from patients suspected of IVC-BSIs.

\section{Materials and Methods}

\section{Hospital setting and study population}

15 The study was carried out in the ICU of the Royal Brisbane and Women's Hospital (RBWH), 16 Queensland, Australia. This is a multi-disciplinary adult ICU that treats all conditions except 17 cardiac surgical and solid organ transplant. Fifteen adult patients (18 years of age or older)

18 with concurrently sited ACs and CVCs and with clinically suspected IVC-BSI were recruited

19 for the study (Table 1). Ethical approval for the study was granted by the RBWH Human

20 Ethics Board and Griffith University Human Research Ethics Committee. Written informed

21 consent was provided by participants or their representatives.

22 IVCs were inserted after skin decontamination with chlorhexidine in alcohol, by experienced

23 ICU medical staff using a Seldinger approach according to accepted guidelines for the

24 prevention of IVC-BSI [11]. All CVCs were ARROWgard Blue ${ }^{\circledR}$ (chlorhexidine acetate and 
1 silver sulfadiazine coated) (Arrow Int, Inc, Reading, PA, USA), and ACs were Vygon Leader

2 Cath brand (Ecouen, France). There was no imposed limitation on dwell time, and resite of

3 catheters always occurred at a new body site. Guide-wire exchange was not performed.

4 Dressings and administration sets were maintained by ICU nurses using unit protocols in

5 accordance with guidelines [11]. If the attending intensive care specialist strongly suspected

6 that the IVC (either AC or CVC) could have been the cause of patient's signs and symptoms,

7 then both IVCs were removed and either replaced at a new site, or an alternative IV access

8 (e.g. peripheral vein) was sought. Diagnosis of IVC-BSI was made using conventional

9 methods [11].

10 IVC samples were cultured using the roll plate technique, and an IVC sample was considered

11 colonised if $>15 \mathrm{cfu}$ (colony forming unit) were isolated [15]. Microorganisms were then

12 isolated and identified according to standard hospital protocol. Data collected included

13 APACHE II score for severity of illness, patient demographics, antimicrobial use, catheter

14 dwell time and reason for removal, ICU and hospital length of stay.

\section{High-throughput sequencing of 16S rRNA}

16 Following processing for culture, catheter tips were suspended in $200 \mu 1$ of lysis buffer,

17 which contained $20 \mathrm{mg} / \mathrm{ml}$ lysozyme, $20 \mathrm{mM}$ Tris- $\mathrm{HCl}(\mathrm{pH} 8.0), 2 \mathrm{mM}$ EDTA, 1.2\% Triton,

18 and Proteinase $\mathrm{K}$ at $37{ }^{\circ} \mathrm{C}$ overnight. Bacterial genomic DNA was extracted from all IVCs

19 using the QIAamp DNA mini kit (Qiagen, Australia). For each catheter, a control (unused)

20 catheter was taken from the original packaging and rolled back and forth on blood agar

21 plates, with bacterial DNA extracted as above. 16S rRNA genes were amplified from purified

22 genomic DNA using the primers F (5' AGA GTT TGA TCC TGG CTC AG 3') and R (5'

23 CTG CTG CCT CCC GTA G 3') which would cover two variable regions (V1 and V2).

24 Thirty different self correction barcodes were designed and added to primers. For each DNA

25 sample, three replicate PCRs were performed. PCR products were cleaned through Qiaquick 
1 PCR Purification kit (Qiagen, Australia). These were sequenced unidirectionally in the

2 reverse direction by means of the Genome Sequencer FLX (GS-FLX) system (Roche,

3 Australia) at 454 Life Sciences.

\section{Bioinformatics}

5 Raw 16S rRNA sequence reads were trimmed as follows. The base quality of reads tends to

6 decrease towards the 3'-prime end. To remove low quality 3'-ends, reads with an average

7 base quality below 25 were trimmed from the 3'-prime end until their average quality score

8 was above 25 . The base quality of reads also considerably decreases after the first ambiguous

9 character ('N') (data not shown). Therefore, reads containing ambiguous characters were

10 additionally trimmed at the occurrence of the first 'N'. Subsequently, low quality reads were

11 identified and excluded using criteria adapted from Huse et al. 2007 [16].

12 All reads were assigned to Bergey's bacterial taxonomy using the RDP classifier and 13 iteratively grouped into Operational Taxonomic Units (OTUs) based on their best BLAST-hit

14 to full-length reference sequences from the RDP database. All reads with a best-BLAST hit 15 with a sequence-identity $>98 \%$, were assigned to OTUs. Subsequently, all remaining reads 16 with a best-BLAST hit, with a sequence-identity $>95 \%$, were then assigned to OTUs. The 17 remaining reads were assigned to OTUs analogously using identity cut-offs of $90 \%$ and $80 \%$.

18 The Chaol microbial richness estimate and overall community diversity (Shannon-Weaver 19 index) were computed from the OTU data. Rarefaction curves were generated by plotting the 20 number of observed genera versus the number of sequences sampled. Coordinates analysis

21 ( $\mathrm{PCoA}$ ) was carried out in $\mathrm{R}$ based on theta-similarities of the relative abundance of the 22 different genera in each sample. Sequences were chimera checked using ChimeraSlayer. 23 Anosim, Adonis and PERMDISP2 were calculated in Calypso. Anosim was run with Jaccard 24 distance as dissimilarity measure and patient as grouping. PERMDISP2 and Adonis were run 
1 for the OTU relative abundance matrix with patients as grouping. The two tailed $t$-Test was

2 used to evaluate the difference between variances.

\section{Genome sequencing of Ralstonia pickettii AU12-08}

4 To maximize the recovery of bacteria, small pieces of IVC samples were cut and washed by

$5 \quad$ PBS and incubated in medium $\mathrm{M}_{10}$ for 24 hours [17]. The solutions were then diluted in a

6 series (neat, 1:10, 1:50, 1:100) on Mueller-Hinton plates. As many different colony types as

7 visually distinguishable, were picked up, purified, and stored in glycerol at $-80^{\circ} \mathrm{c}$. Bacterial

8 DNA was released from bacterial cells by boiling, and one microliter was used as a template

9 in PCR amplification. Purified PCR products were then sequenced, and compared to NCBI

10 GenBank database using BLAST program. The most predominant bacterial species identified

11 in pyrosequencing were chosen for further study.

12 Bacterial metagenomic DNA from Ralstonia pickettii AU12-08 was extracted and genome

13 sequence of bacterial species was determined on the Genome Sequencer FLX (GS-FLX)

14 system (Roche, Australia) at 454 Life Sciences. Metagenomic contigs were de novo

15 assembled using GS De Novo assembler (version 2.3; Roche). Automatic genome annotation

16 was performed on the RAST server [18] and IMG/M-ER [19].

\section{Biofilm growth essay}

18 The assay to grow and quantitate biofilms has been used previously for other bacterial species

19 [20,21]. An overnight broth culture was diluted 1:200 in fresh broth and $200 \mu$ was

20 inoculated into the wells of a 96 well Linbro tissue culture plate. The plates were incubated at

$2137^{\circ} \mathrm{C}$, under $5 \% \mathrm{CO}_{2}$, for 12 days. Growth was then assessed by measuring the optical density

22 at $490 \mathrm{~nm}\left(\mathrm{OD}_{490}\right)$ in a BioRad plate reader. To quantitate biofilm formation, $20 \mu$ l of Difco

23 crystal violet (Becton Dickinson, Australia) was added to each well and incubated at room

24 temperature for 15 minutes. Wells were washed vigorously with distilled water and the plate 
1 was air dried. A volume of $230 \mu \mathrm{l}$ of $95 \%$ ethanol was added to each well and the $\mathrm{OD}_{570}$ was

2 measured. All strains were tested in quadruplicate. Each plate included 4 wells, which

3 contained sterile broth instead of bacteria, but were treated identically otherwise. The $\mathrm{OD}_{570}$

4 was standardized against these wells.

\section{Accession number}

6 The draft genome of Ralstonia rickettii AU12-08 has been deposited at GenBank under

7 accession number ASZV 00000000.

\section{Results}

\section{Sample characteristics}

10 The 15 recruited patients had a mean age of 51.3 years, an APACHE II score of 23.6, ICU

11 stay of 16.7 days, and $80 \%$ were on systemic antimicrobials at the time of catheter removal

12 (Table 1). The mean duration of catheter placement was 5.6 days, and there was no difference

13 in dwell time between ACs and CVCs ( $p>0.1$, two tailed $t$-Test).

14 According to the results of semi-quantitative cultures, 4 of the 30 removed IVCs were

15 considered colonised, and in these cases both the AC and CVC were colonised from two 16 patients. Isolates are including Staphylococcus epidermidis (catheter numbers 2A and 2C),

17 Staphylococcus hominis (3A), and mixed enteric and skin bacteria (3C) (Table 1). One of the

$1815(6.66 \%)$ patients was diagnosed as IVC-BSI (matched tip and blood cultures with no other

19 explanatory source for the symptoms). Both colonisation (semi-quantitative method) and

20 IVC-BSI occurred in patients already receiving multiple antibiotics (see Table 1).

\section{Bacterial community profiles of IVCs}

22 No bacterial DNA was detected or amplified from negative control IVCs. These control

23 results indicate that the contribution to the bacterial community from manufacture of IVCs,

24 DNA extraction procedures and PCR reagents was negligible. Bacterial DNA was extracted 
1 from each IVC sample and individually amplified. A total of 50,364 sequences (18.8\%) did

2 not meet the quality control criteria and, thus, were excluded, and 217,488 high-quality

3 sequence reads were used for further analysis. Chimera checks showed that sequences were

4 not chimeric. Overall, microbial communities on both types of IVCs had a surprisingly high

5 diversity and complex community structure. 16S rRNA gene sequences were assigned to

6 sixteen bacterial phyla: Acidobacteria, Actinobacteria, Bacteroidetes, Cyanobacteria,

7 Deinococcus-Thermus, Firmicutes, Fusobacteria, Gemmatimonadetes, Nitrospirae, OD1,

8 Proteobacteria, Spirochaetes, Tenericutes, Thermotogae, TM7 and unclassified bacteria (Fig.

9 1). The most dominant phylum was Proteobacteria ( $79.8 \%$ of $16 \mathrm{~S}$ rRNA reads), which

10 included the taxonomic genus Ralstonia (53\%), Escherichia group (16\%), and

11 Bradyrhizobium (2\%). The term of Escherichia group used in this study since the16S rRNA

12 has difficulty on differentiating Escherichia and Shigella, and it was also suggested that

13 Escherichia and Shigella were sufficiently similar for placement in a single genus [22]. A

14 high number of OTUs was observed for each sample, ranging from 57 to 168 (Fig. 2). The

15 number of different genera per sample (bacterial richness) ranged from 22 to 59, and the

16 overall diversity (Shannon-Weaver index) was in the range of 3.09 to 4.08 .

17 Microbial communities on ACs and CVCs had a similar community composition and 18 diversity. Ralstonia were dominant and accounted for $52.1 \%$ of examined sequences in ACs, 19 followed by Escherichia group (14.5\%), Staphylococcus (5.2\%), and Propionibacterium 20 (5.3\%). CVCs had a similar community structure at an OTU level. Over $90 \%$ of identified

21 OTUs were present on both ACs and CVCs. Further, in terms of overall microbial diversity, 22 no difference was found between AC and CVC samples - the Shannon-Weaver diversity 23 index values for both ACs and CVCs were around 3.5 ( $p>0.05$, two tailed $t$-Test). All results 24 indicated no statistically significant differences in the bacterial communities examined from 25 CVCs and ACs ( $>00.05$, two tailed t-Test). The PCoA (Fig. 3a) suggested that bacterial 
1 communities on ACs and CVCs had similar community profiles, as the AC and CVC

2 microbial communities did not form distinct clusters. Thus, incidence of bacterial

3 colonisation and, therefore, the potential for causing IVC-BSI did not appear to differ

4 between ACs and CVCs. These findings support the assertion that ACs should be considered

5 as an equally likely site, as CVCs, of catheter colonization in critically ill patients [12].

6 For the majority of patients (11/15) the microbial communities found on the two different

7 catheters were more similar than the average distance of microbial communities from

8 different patients. The average Jaccard distance of communities from different patients was

90.6 , the average Jaccard distance of communities from the same patient was $0.39(\mathrm{p}=0.12$, $\mathrm{t}$ -

10 test) (Fig. 4). According to Anosim, Adonis and PERMDISP2, the intra-patient communities

11 were generally more similar than between-patient communities (Anosim: $p=0.002$; Adonis:

$12 \mathrm{p}=0.001$; PERMDISP2: 1e-225).

13 We compared the bacterial communities present on 'colonised' and 'uncolonised' IVCs. The

14 dominant bacteria on both groups were Ralstonia and Escherichia group. No statistically

15 significant differences were found between these two groups in terms of bacterial profiles,

16 OTU distribution, Shannon-Weaver diversity, and PCoA (Fig. 3b). Moreover, it was also

17 noticed that for patient number 2, who had both IVCs 'colonised' by coagulase-negative

18 staphylococcus (CNS) and Staphylococcus spp. using the roll-plate culture method,

19 Staphylococcus spp. in fact represented only $3 \%$ of the bacterial community present on both

20 IVCs via molecular methods. Further, for patient number 15, the AC and CVC yielded sub-

21 diagnostic growth $(<15$ cfu) of Staphylococcus spp., and for mixed Staphylococcus and

22 Escherichia group respectively, whereas over $60 \%$ of $16 \mathrm{~S}$ rRNA sequences from the AC

23 were Staphylococcus spp. and $72 \%$ of $16 \mathrm{~S}$ rRNA gene sequences from CVC were

24 Acinetobacter spp. Bacterial communities on this patient's IVCs were different from the 
1 remaining patients' IVCs, which were dominated by species closely related to Ralstonia

2 and/or Escherichia group.

3 Eight IVC samples (4 ACs and 4 CVCs) came from four patients not receiving antibiotic

4 treatment. We compared the bacterial communities on these samples to the 22 IVC samples

5 from 11 patients treated with intravenous antibiotics in the two weeks prior to sample

6 collection. Statistically, there was no significant difference in the bacterial communities on

7 IVCs from patients with, or without, antibiotic treatment, as confirmed by the results of

8 PCoA (Fig. 3c) and OTU distribution.

\section{$9 \quad$ Ralstonia pickettii AU12-08 genome sequencing}

10 The closest cultured relative of AU12-08 is $R$. pickettii, and it shares $100 \%$ sequence identity

11 with the 16S rRNA genes of the predominant bacterial species in pyrosequencing libraries.

12 Therefore, a thorough understanding of $R$. pickettii AU12-08 genome is vital for the

13 systematic understanding of bacterial pathogenesis on IVC-related infections. The sequence

14 data consists of $6,229,152$ bp chromosome with $\mathrm{G}+\mathrm{C}$ content of $63.6 \%$. The $R$. pickettii

15 AU12-08 genome harbours 50 tRNA genes coding for all amino acids, and 5733 predicted

16 protein coding genes consistent with other sequenced Ralstonia spp. [23,24]. Comparisons of

17 the general genomic features of $R$. pickettii AU12-08 with nosocomial pathogenic $R$. pickettii

18 strains $12 \mathrm{~J}$ and $12 \mathrm{D}$, and environmental isolate $R$. solanacearum GM1000, are shown in

19 Table 2.

20 A seven gene operon coding for Pel synthesis machinery was present in R. pickettii AU12-08.

21 Pel was originally found to be responsible for the production of glucose-rich biofilm matrix 22 exopolysaccharide in strain Pseudomonas aeruginosa 14 [25]. The detailed comparison of 23 Pel coded by $R$. pickettii AU12-08 genome with R. pickettii 12J, R. pickettii 12 D and PA 14, 24 showed a high degree of similarity with respect to gene organization and predicted function 
1 (Fig. 1Sa). An independent study by Vasseur et al. showed that glucose-rich polysaccharides

2 are essential for the formation of a surface-associated biofilm; thus, it is a major component

3 of biofilm in strain Pseudomonas aeruginosa K [26]. The pel operon contains seven genes,

4 pelA to pelG, which display sequence similarity with genes that encode sugar-processing

5 enzymes. These include oligogalacturonide lyase (pelA), glycosyltransferases (pelC and

6 pelF), sucrose synthase (pelE), and transmembrane proteins (pelD and pelG). Transposon

7 insertion into, or deletion of, these genes resulted in severe defects in biofilm formation [26].

8 More genes in the $R$. pickettii AU12-08 genome were found to encode resistance to

9 antibiotics and toxic compounds compared to $R$. pickettii 12J, R. pickettii 12D and $R$.

10 solanacearum GM1000 (table 2). The R. pickettii AU12-08 genome contains 22 putative

11 Multidrug Resistance Efflux Pumps (resistance nodulation division family), which enable

12 bacteria to excrete antibiotics and other toxic compounds. Multidrug Resistance Efflux

13 Pumps have been previously demonstrated to not only confer resistance to drugs used in

14 therapy, but also to have a role in bacterial pathogenicity, i.e., through bacterial colonization

15 and bacterial survival in the host [27]. Seven genes in chromosome 1, and 3 genes in

16 chromosome 2 of $R$. pickettii AU12-08 genome, were coding for beta-lactamase including

17 beta-lactamase (EC 3.5.2.6), beta-lactamase class $\mathrm{C}$, and penicillin binding proteins and beta-

18 lactamase class D. Environmental isolate R. solanacearum GM1000 does not encode beta-

19 lactamase (EC 3.5.2.6), beta-lactamase class $\mathrm{C}$ or penicillin binding proteins. $R$. pickettii

20 AU12-08 shares a high similarity with PA14 on beta-lactamase which has been reported to

21 resist Beta-lactam antibiotics (Fig. 1Sb). In addition, the presentation of gyrA, gyrB, parC

22 and parE in $R$. pickettii AU12-08 might indicate the resistance of fluoroquinolones. Thirteen

23 genes were coded for components of tripartite multidrug resistance system. Further, 170

24 genes were coded for resistance toxic compounds, including cobalt-zinc-cadmium, copper

25 homeostasis, mercury operon, arsenic and bile hydrolysis. 


\section{Biofilm essay}

2 R. pickettii AU12-08 recovered from IVC tips was studied for biofilm formation. To

3 characterise the kinetics of biofilm formation, the levels of biofilm formation were measured

4 after 3, 6, 9 and 12 days respectively. We also examined the effect of other bacterial species

5 (E. coli and S. aureus) on the general growth characteristics of R. pickettii AU12-08 (Fig. 5a).

6 We compared the biofilm formation of R. pickettii AU12-08 to that of other bacterial species

7 by measuring the $\mathrm{OD}_{570}$. As shown in Fig. 4, throughout duration of the biofilm formation,

8 the biofilm formed by $R$. pickettii AU12-08 alone, was less than that when formed in

9 combination with multiple bacterial species (Fig. 5b). These results suggest that $R$. pickettii

10 AU12-08 forms biofilms independently, and also in a greater volume of biofilms when

11 present with other bacterial species.

\section{Discussion}

13 Using high-throughput 16S rRNA sequencing, we demonstrated the complex diversity of the 14 microbiological flora on commonly used intravascular catheters. An understanding of the 15 composition of bacterial communities on IVCs is essential for the prevention and treatment of 16 IVC-BSI. Many identified bacterial species in this study were known pathogens or 17 opportunistic pathogens such as Ralstonia spp., Escherichia group spp., Staphylococcus spp., 18 Streptococcus spp., Acinetobacter spp., Chryseobacterium meningosepticum, 19 Corynebacterium spp., Stenotrophomonas spp. and Pseudomonas spp.

20 The predominant bacterial species on examined IVCs were closely related to $R$. pickettii 21 (over 50\% of examined sequences). R. pickettii (Pseudomonas pickettii) is a non-

22 fermentative, gram-negative bacterium isolated from environmental and clinical samples. $R$.

23 pickettii has the ability to survive in $0.05 \%$ chlorhexidine solutions [28] and penetrate $0.2 \mathrm{~mm}$

24 filters [29]. Therefore, many of the cases of infection with $R$. pickettii were due to 25 contaminated water or aqueous solutions given intravenously [30]. Even "sterile" medical 
1 products including ranitidine and saline solution had been reported to be contaminated with

2 R. pickettii [31,32]. Several nosocomial catheter-related outbreaks have been described $3 \quad[30,33,34]$.

4 R. pickettii growth is slow and unfavourable on the blood agar plates currently used for 5 routine IVC culture, which may explain why clinical results are generally negative for this 6 organism. Our results challenge the belief that $R$. pickettii is of low virulence and incidence in

7 IVCs, and suggest that it is, in fact, embedded in a biofilm and thus escaping detection using 8 current methods. It may be of virulence itself, or it may be important in the subsequent 9 colonisation or activity of other microbes. R. pickettii AU12-08 contains genes important for 10 biofilm formation. A seven gene operon coded for Pel synthesis machinery. Pel was 11 originally found to produce glucose-rich biofilm matrix exopolysaccharide in Pseudomonas 12 aeruginosa 14 . The biofilm assay used in this study demonstrated that $R$. pickettii AU12-08 is 13 capability of forming biofilms.

14 Patients recruited within this study were treated with vancomycin, meropenem and 15 piperacillin/tazobactam (Table 1). Vancomycin is used to treat infections caused by gram16 positive bacteria, meropenem is a beta-lactam and piperacillin/tazobactam combines the 17 extended-spectrum penicillin piperacillin and $\beta$-lactamase inhibitor tazobactam. The genome 18 of R. pickettii AU12-08 harboured numerous genes coding for resistance of antibiotics and 19 toxic compounds. $R$. pickettii AU12-08 has 22 putative multidrug resistance efflux pumps to 20 enable bacteria to excrete antibiotics and toxic compounds in catheter coatings. $R$. pickettii 21 AU12-08 are gram-negative bacteria, therefore they are not sensitive to vancomycin. $R$. 22 pickettii AU12-08 also contains $\beta$-lactamase, which can break the $\beta$-lactam (meropenem)

23 structure. $R$. pickettii AU12-08 possesses genes encoding proteins involved in biofilm 24 formation, that also contribute to increased antimicrobial drug resistance. Considering that 25 most patients were receiving antibiotic treatment, yet most IVCs had R. pickettii present, it 
1 would appear that it is able to resist current therapy. Whilst the origin of the detected bacteria

2 cannot be ascertained from our study, it is possible that some organisms may have been

3 environmentally acquired. Since all controls were negative, it is unlikely that contamination

4 occurred in the laboratory. Our study did not include specimens from patients who were not

5 suspected of infection at the time of catheter removal, and this could be a focus of future

6 studies, as long as the dwell time of catheters was matched between groups. Colonization of

7 the indwelling IVC can occur via health care workers hands if they contaminate the hub or

8 infusates while giving medications and fluids, or when drawing blood. This is plausible since

9 these IVCs are typically accessed for therapy many times per day - staff hand hygiene and

10 administration set decontamination prior to access may not always be optimal. Other

11 organisms we identified may be resident on patients' skin - gram positives but also gram-

12 negative bacteria, which increase in density on the skin and heightened the increased acuity

13 of illness and duration of hospitalization typical in ICU patients. To verify with certainty the

14 source of these bacteria would require a comprehensive assessment beyond the scope of the

15 current study.

\section{Conclusions}

17 This study demonstrates the utility of high-throughput sequencing for examining bacterial 18 communities on medical devices. Many microorganisms that were encountered were closely

19 related to known human opportunistic or true pathogens and some have been commonly

20 involved in IVC-related infections. Description of these bacterial communities will improve

21 our understanding of IVC-related infections since bacterial colonization is the first step

22 leading to IVC-related infections. In addition, better understanding of these bacterial

23 communities may aid the development of more efficient diagnostic and therapeutic

24 approaches for IVC-related infections, both of which remain difficult in modern medicine. 


\section{Acknowledgments}

3 We thank Jenny Murfield for editorial assistance with the manuscript. Dr. Li Zhang is

4 supported by Australian National Health and Medical Research Council Training Clinical

5 Research Fellowship, Australian Government (grant number 597491). The research was

6 supported by project grants from Griffith Health Institute and Health Practice Innovation

7 (Griffith University, Australia.

\section{Conflict of Interest}

$9 \quad$ All authors declare no conflicts of interest relevant to this article.

10 
2 Table 1. Patient demographics and roll-plate intravascular catheters culture results.

\begin{tabular}{|c|c|c|c|c|c|}
\hline $\begin{array}{l}\text { Patient } \\
\text { number }\end{array}$ & $\begin{array}{l}\text { ICU } \\
\text { stay } \\
\text { (days) }\end{array}$ & Diagnosis & $\begin{array}{l}\text { APACHE } \\
\text { II score }\end{array}$ & Antibiotic usage & 'Roll-plate' culture result \\
\hline 1 & 14 & Subdural hematoma & 27 & Meropenem and Vancomycin & $\mathrm{NG}$ \\
\hline 2 & 17 & Sepsis pneumonia & 27 & $\begin{array}{c}\text { Vancomycin, Piperacillin and } \\
\text { Tazobactam }\end{array}$ & $\begin{array}{l}\text { 2A: } 100 \text { cfu Staphylococcus } \\
\text { epidermidis } \\
\text { 2C: } 10^{3} \text { cfu mixed CNS }\end{array}$ \\
\hline 3 & 12 & Peritonitis & 22 & Piperacillin and Tazobactam & $\begin{array}{l}\text { 3A: } 50-100 \text { cfu Staphylococcus } \\
\text { hominis } \\
\text { 3C: } 50-100 \text { cfu mixed } \\
\text { Staphylococcus and Escherichia }\end{array}$ \\
\hline 4 & 8 & Cardiac arrest & 29 & None & NG \\
\hline 5 & 7 & Sepsis & 18 & None & NG \\
\hline 6 & 9 & Respiratory failure & 19 & None & NG \\
\hline 7 & 14 & Subdural hematoma & 27 & None & NG \\
\hline 8 & 38 & Respiratory failure & 30 & Piperacillin and Tazobactam & NG \\
\hline 9 & 10 & Burn & 16 & Piperacillin and Tazobactam & NG \\
\hline 10 & 8 & Fecal peritonitis & 13 & Meropenem and Vancomycin, & NG \\
\hline 11 & 32 & $\begin{array}{l}\text { Subarachnoid } \\
\text { hemorrhage }\end{array}$ & 19 & $\begin{array}{l}\text { Vancomycin, Meropenem, } \\
\text { Piperacillin and Tazobactam, }\end{array}$ & NG \\
\hline 12 & 12 & Craniotomy & 30 & $\begin{array}{c}\text { Meropenem, Piperacillin and } \\
\text { Tazobactam }\end{array}$ & NG \\
\hline 13 & 38 & Multi-trauma & 21 & Piperacillin and Tazobactam & NG \\
\hline 14 & 19 & Cardiac arrest & 36 & Piperacillin and Tazobactam & NG \\
\hline 15 & 13 & Liver failure & 20 & $\begin{array}{l}\text { Meropenem, Vancomycin, } \\
\text { Piperacillin and Tazobactam, }\end{array}$ & NG \\
\hline
\end{tabular}


1 Table 2. General features of genomes of $R$. pickettii strains AU12-08, 12J, $12 \mathrm{D}$ and $R$. 2 solanacearum GM1000.

\begin{tabular}{|c|c|c|c|c|}
\hline & $\begin{array}{c}\text { R. pickettii } \\
\text { AU12-08 }\end{array}$ & $\begin{array}{c}\text { R. pickettii } \\
12 \mathrm{~J}\end{array}$ & $\begin{array}{c}\text { R. pickettii } \\
\text { 12D }\end{array}$ & $\begin{array}{c}\text { R. solanacearum } \\
\text { GM1000 }\end{array}$ \\
\hline Origin & Clinical isolate & $\begin{array}{l}\text { Nosocomial } \\
\text { pathogen }\end{array}$ & $\begin{array}{l}\text { Nosocomial } \\
\text { pathogen }\end{array}$ & $\begin{array}{l}\text { Environmental } \\
\text { isolate }\end{array}$ \\
\hline Length (Mb) & 6.23 & 5.33 & 5.69 & 5.81 \\
\hline GC\% & 63.7 & 63.6 & 63.3 & 67 \\
\hline \#CDs & 5733 & 5100 & 5361 & 5635 \\
\hline tRNA & 50 & 57 & 54 & 57 \\
\hline \multicolumn{5}{|l|}{ Virulence factors } \\
\hline $\begin{array}{l}\text { Multidrug } \\
\text { resistance efflux } \\
\text { pumps }\end{array}$ & 22 & 18 & 18 & 13 \\
\hline Beta-lactamase & 10 & 4 & 4 & 3 \\
\hline $\begin{array}{l}\text { Multidrug } \\
\text { resistance } \\
\text { tripartite systems }\end{array}$ & 13 & 3 & 3 & 11 \\
\hline $\begin{array}{l}\text { Resistance to } \\
\text { fluoroguinolones }\end{array}$ & 4 & 4 & 5 & 4 \\
\hline $\begin{array}{l}\text { Resistance to toxic } \\
\text { compounds }\end{array}$ & 168 & 128 & 112 & 55 \\
\hline $\begin{array}{l}\text { Quorum sensing } \\
\text { and biofilm } \\
\text { formation }\end{array}$ & 9 & 12 & 13 & 12 \\
\hline
\end{tabular}

3 


\section{Figure Legends}

2 Fig. 1. Microbial genera detected in intravascular catheters. A represent arterial catheters and $\mathrm{C}$ represent central venous catheters.

Fig. 2. Heat map depicting relative abundance of bacterial genera across intravascular catheters samples of 15 patients. A represent arterial catheters and C represent central venous catheters.

Fig. 3. Principal coordinate analysis of bacterial communities of intravascular catheters samples based on UniFrac distances. (a) 15 samples; (b) colonised IVCs (grey balls) and uncolonised IVCs (black balls); (c) antibiotic treatment (red balls) and without antibiotic treatment (blue balls). Each point corresponds to each IVC sample.

Fig. 4. Jaccard distance measuring dissimilarity between and intra 15 patients.

Fig. 5. (a) Time course of biofilm formation of five groups of bacteria. (b) Biofilm formation of five groups of bacteria. The data represent the average absorbance at $570 \mathrm{~nm}$ ( \pm s.e.m.). A, Ralstonia pickettii; B, Pseudomonas aeruginosa; C, Escherichia coli; D, Staphylococcus aureus; E, Ralstonia pickettii together with Escherichia coli and Staphylococcus aureus.

Fig. 1S. (a) Comparison of extracellular matrix proteins (Pel) maps reconstructed from R. pickettii AU 12-08 and other five bacterial strains. 1, Pel B; 2, Pel C; 3, Pel A; 4, Pel D; 5, Pel E (987 bp); 6, Pel F; 7, Pel G. (b) Beta-lactamase from R. pickettii AU 12-08. (c) Multiple drug efflux pumps from R. pickettii AU 12-08. 
Fig. 1.

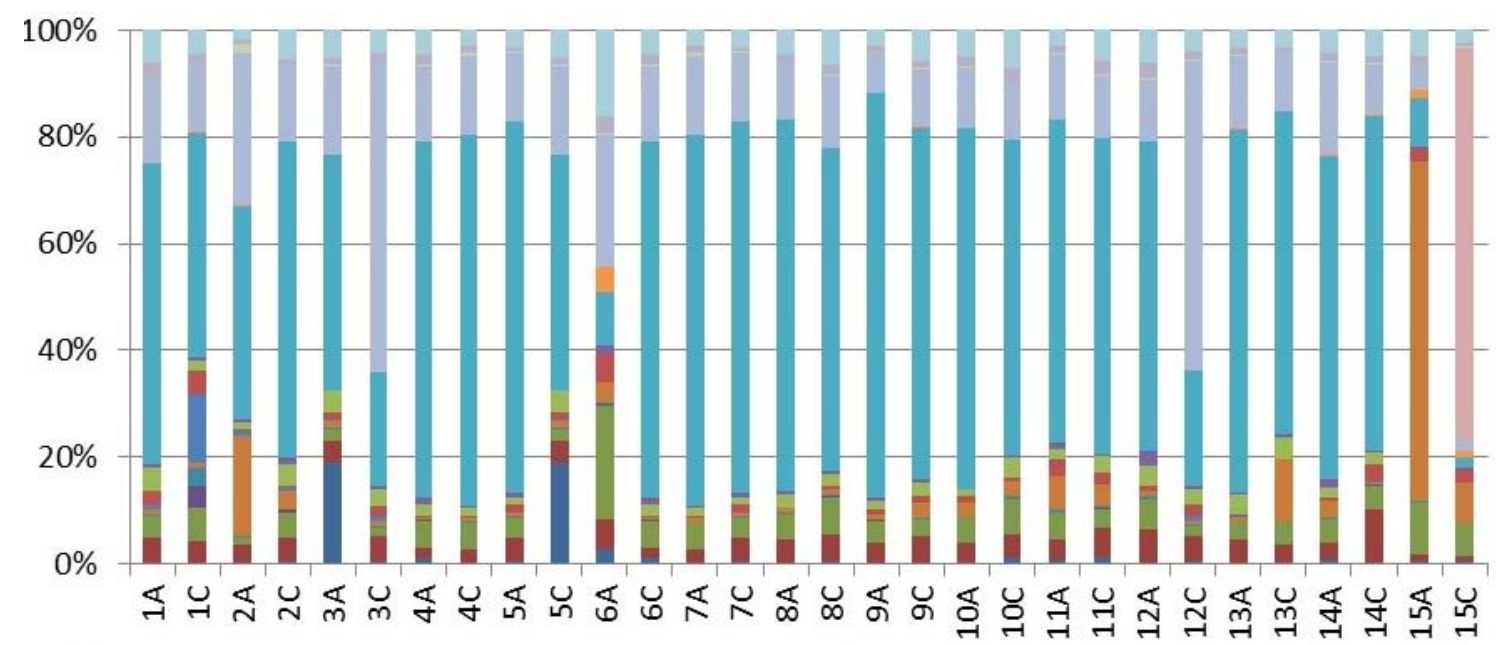

- Actinobacteria Corynebacterium

- Actinobacteria Propionibacterium

- Cyanobacteria Streptophyta

Bacilli Aerococcus

- Alphaproteobacteria Bradyrhizobium

- Betaproteobacteria Ralstonia

- Gammaproteobacteria Escherichia group

- Gammaproteobacteria Stenotrophomonas

2

nemaining actinobacteria Rhodococcus

- Flavobacteria Chryseobacterium

- Bacilli Staphylococcus

- Bacilli Streptococcus

- Alphaproteobacteria Novosphingobium

- Betaproteobacteria Methylophilus

- Gammaproteobacteria Acinetobacter

- Unclassified Bacteria

3 
Fig. 2.

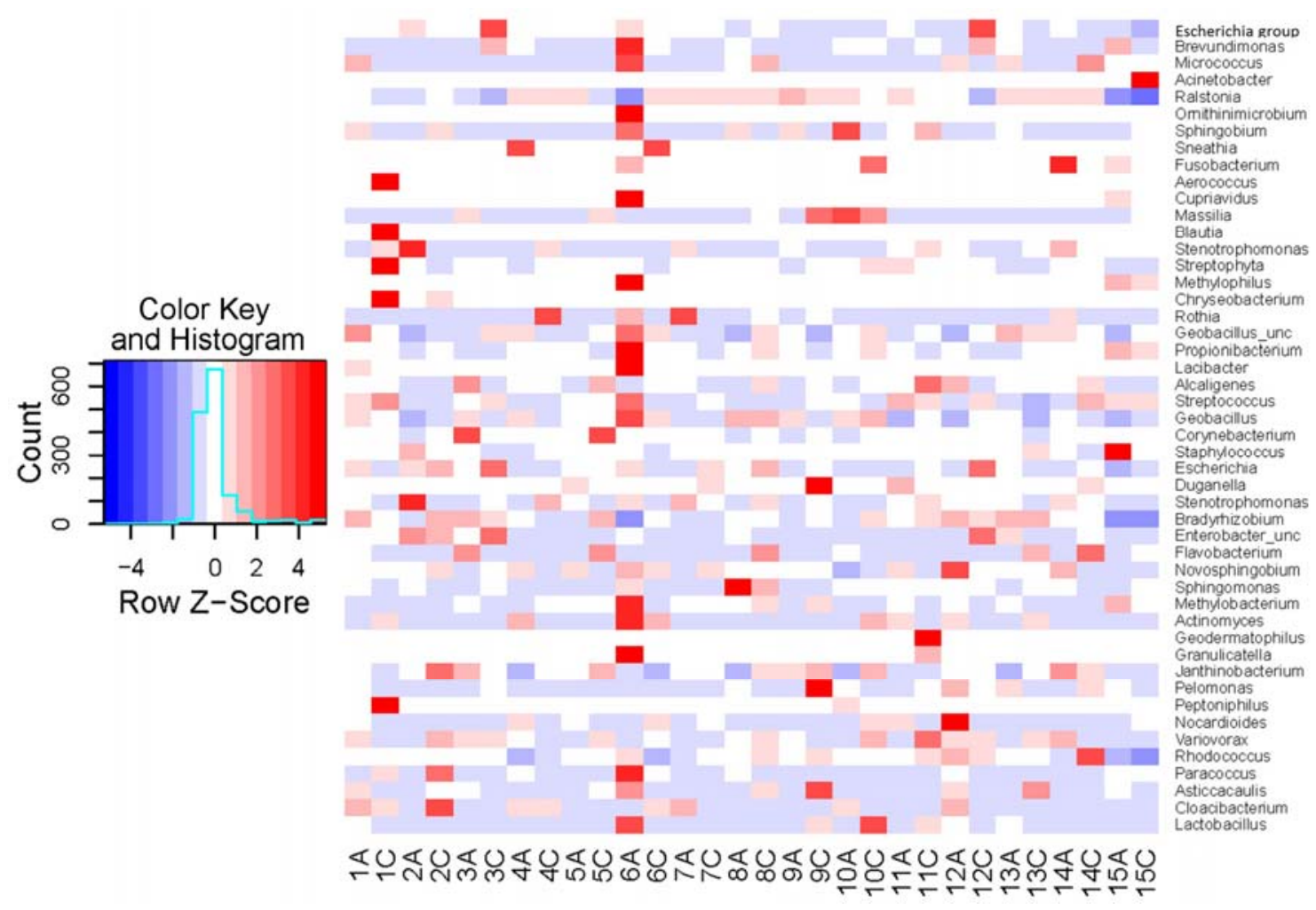


$1 \quad$ Fig. 3.

2

(a)

3

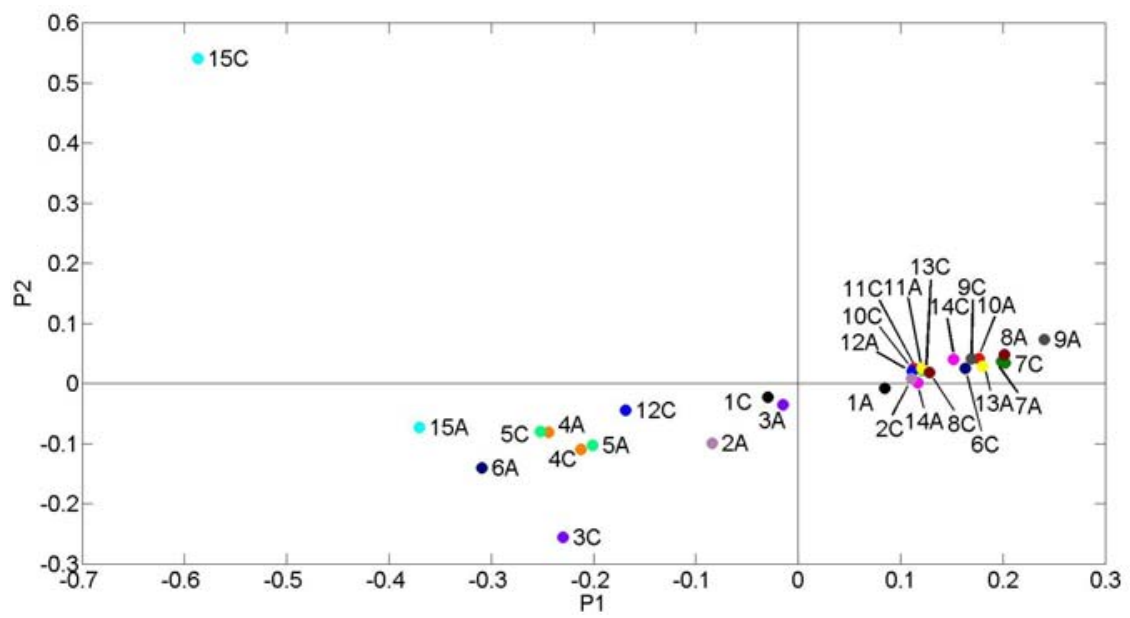

4

(b)

5

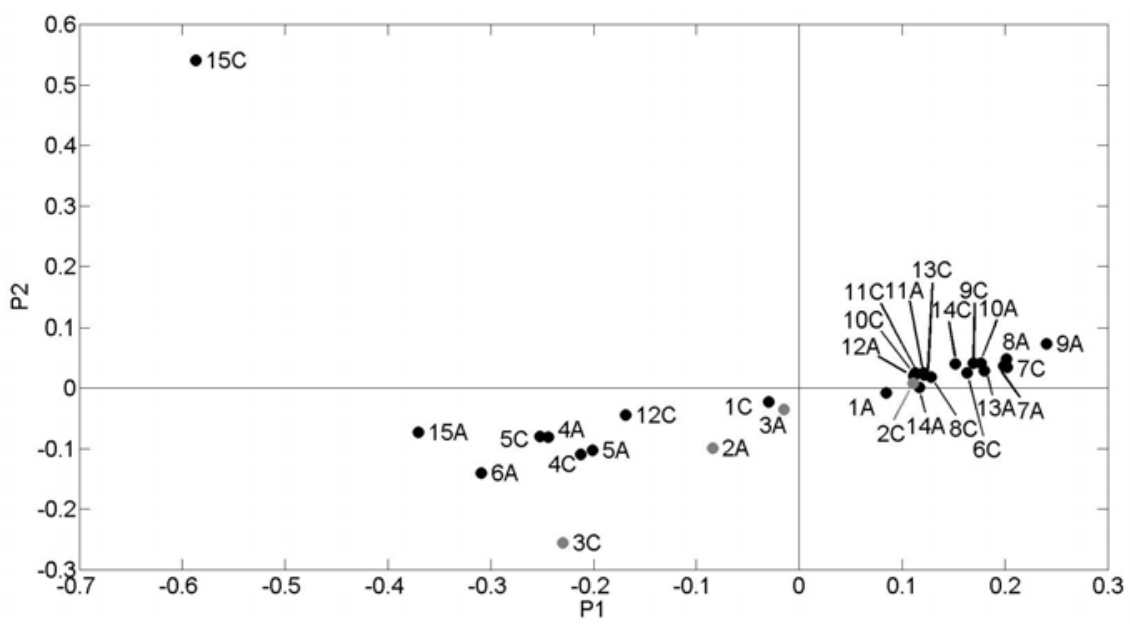

6 
1 (c)

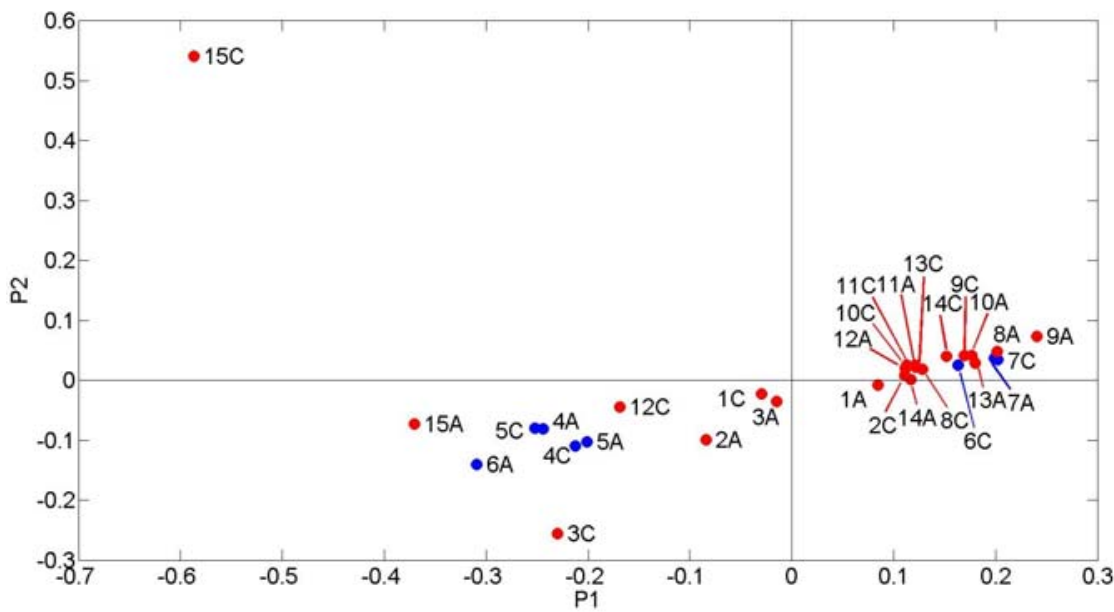


$1 \quad$ Fig. 4.
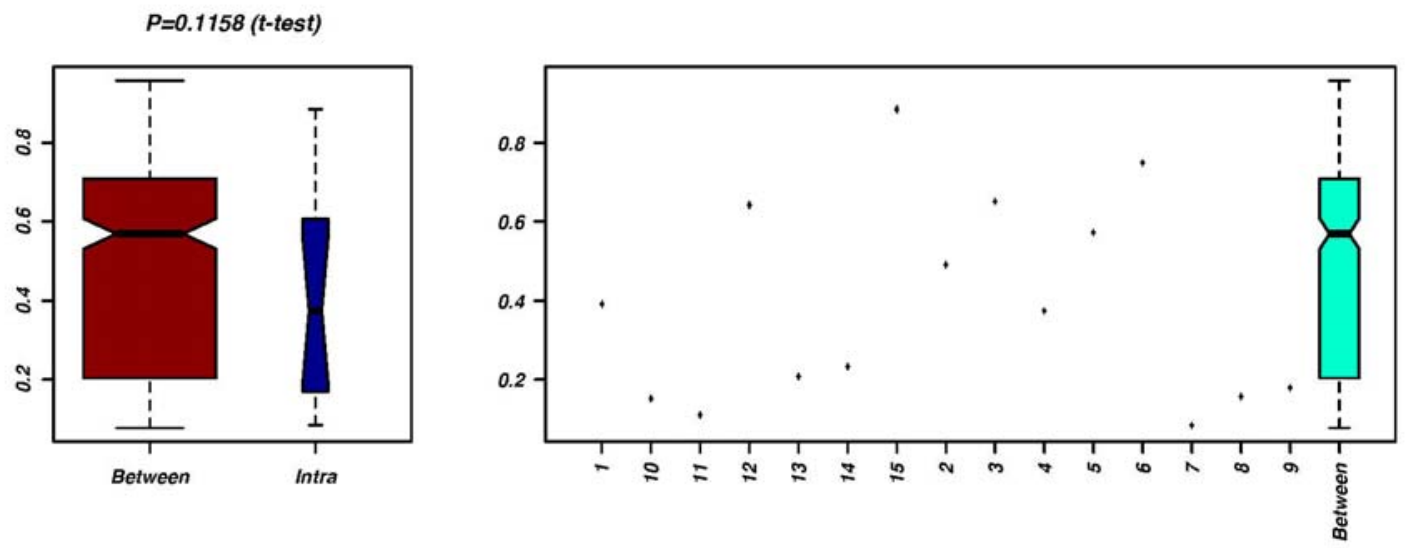

2 
1 Fig. 5.

2

(a)

3

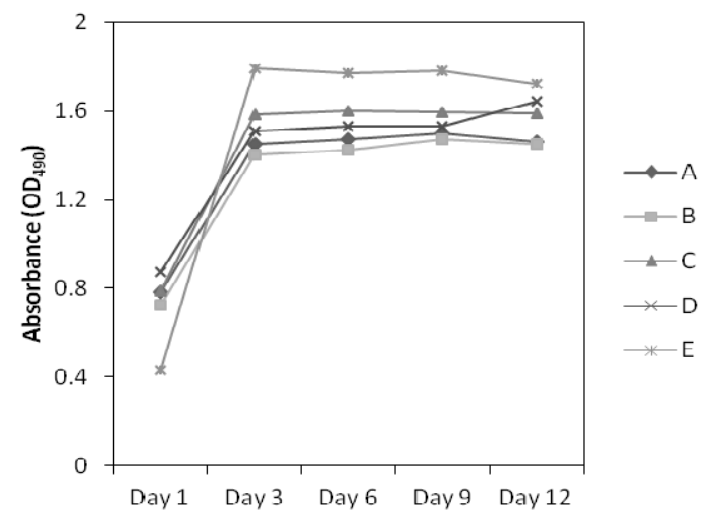

4 (b)

5

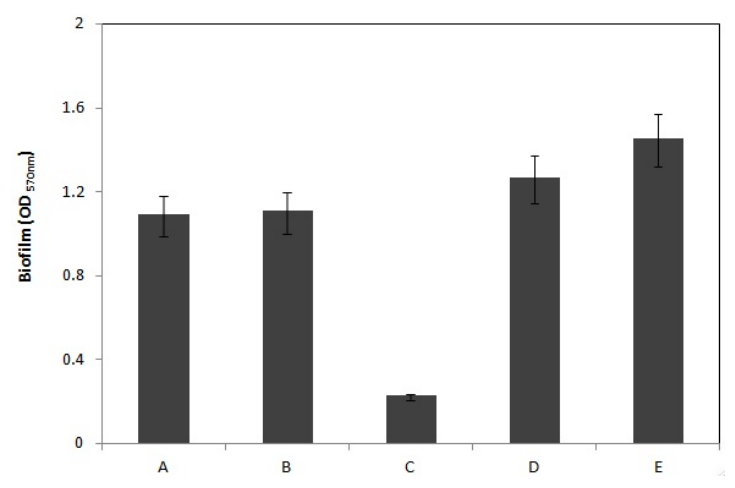

6

7 
$1 \quad$ Fig. 1S.

2 (a)

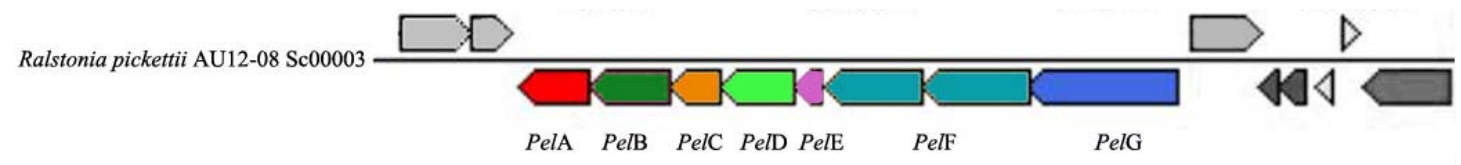

Ralstonia pickettii 12D chromosome 1
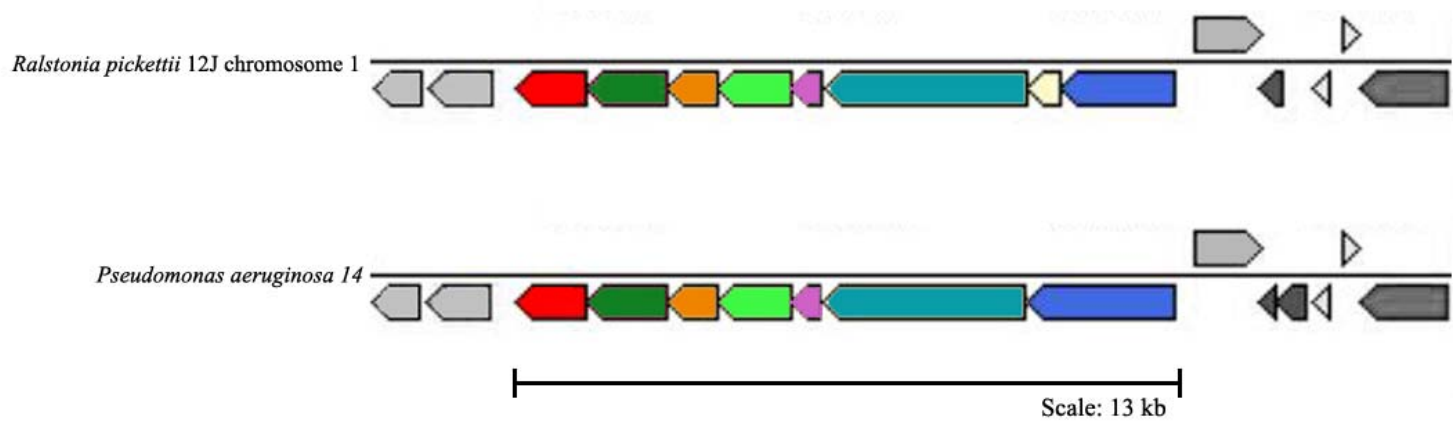
(b)
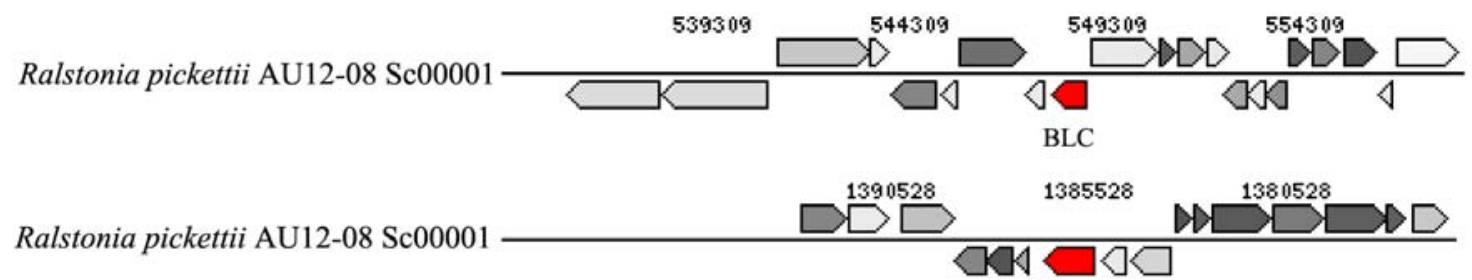

BLC

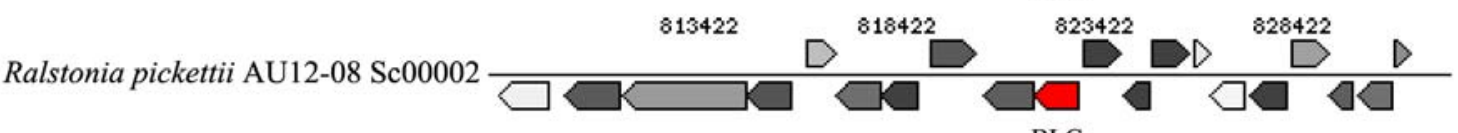

BLC
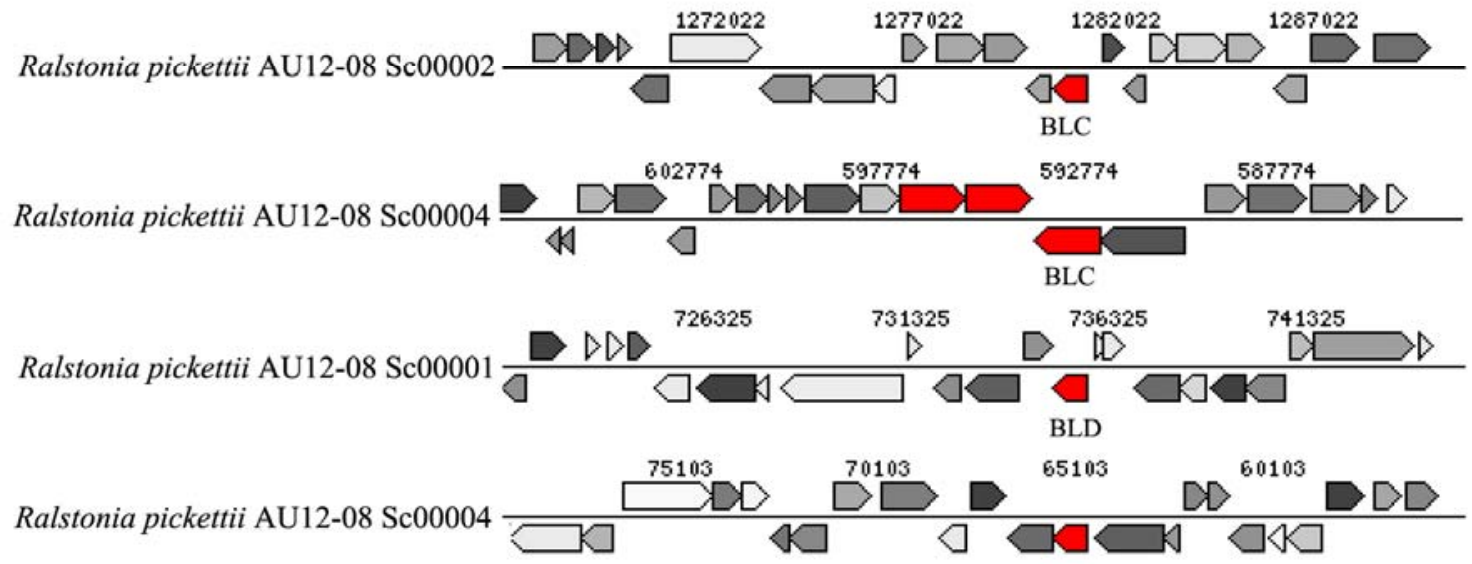

BLD

3 BLC represents beta-lactmase class C and penicillin binding protein; BLD represents beta-lactmase class D. 


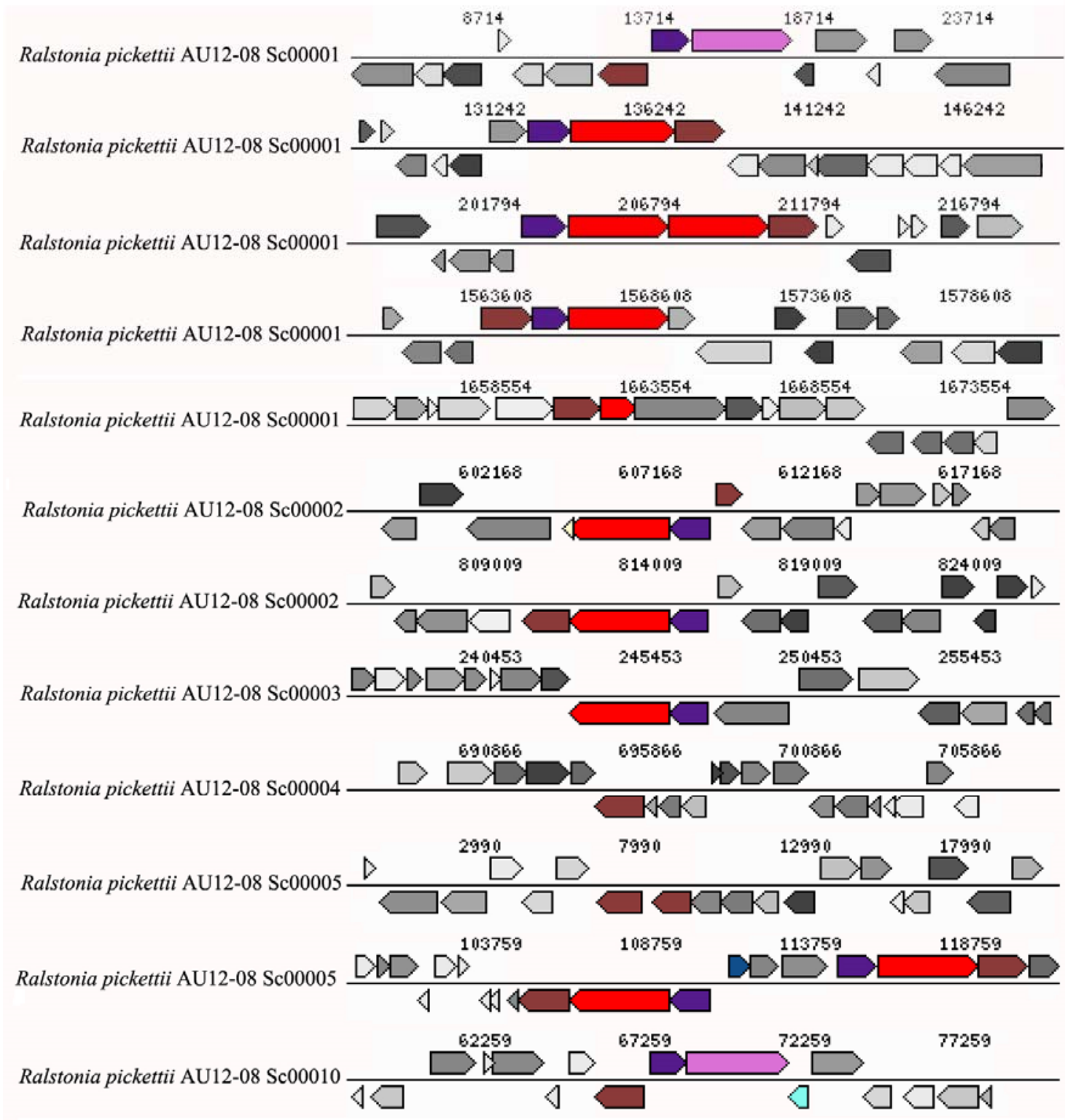


1. Mermel LA, Allon M, Bouza E, Craven DE, Flynn P, O'Grady NP, Raad, II, Rijnders BJ, Sherertz RJ, Warren DK (2009) Clinical practice guidelines for the diagnosis and management of intravascular catheter-related infection: 2009 Update by the Infectious Diseases Society of America. Clin Infect Dis 49 (1):1-45. doi:10.1086/599376

2. Allegranzi B, Bagheri Nejad S, Combescure C, Graafmans W, Attar H, Donaldson L, Pittet D (2011) Burden of endemic health-care-associated infection in developing countries: systematic review and meta-analysis. Lancet 377 (9761):228-241. doi:S0140-6736(10)614584 [pii] 10.1016/S0140-6736(10)61458-4

3. Gogos CA, Leonidou L (2010) Catheter-related bloodstream infections: catheter management according to pathogen. Int $J$ Antimicrob Agents 36:S26-S32. doi:10.1016/j.ijantimicag.2010.11.004

4. Timsit JF (2007) Diagnosis and prevention of catheter-related infections. Current Opinion in Critical Care 13 (5):563-571

5. Zhang L, Xu ZH (2008) Assessing bacterial diversity in soil. Journal of Soils and Sediments $8(6): 379-388$

6. Zhang L, Sriprakash KS, McMillan D, Gowardman JR, Patel B, Rickard CM (2010) Microbiological pattern of arterial catheters in the intensive care unit. BMC Microbiology 10:266-275

7. Rogers GB, Carroll MP, Bruce KD (2009) Studying bacterial infections through cultureindependent approaches. J Med Microbiol 58 (Pt 11):1401-1418. doi:jmm.0.013334-0 [pii] 10.1099/jmm.0.013334-0

8. Mancini N, Carletti S, Ghidoli N, Cichero P, Burioni R, Clementi M (2010) The Era of Molecular and Other Non-Culture-Based Methods in Diagnosis of Sepsis. Clinical Microbiology Reviews 23 (1):235-+. doi:10.1128/Cmr.00043-09

9. Pratt RJ, Petlowe CM, Wilson JA, Loveday HP, Harper PJ, Jones SRLJ, McDougall C, Wilcox MH (2007) epic2: National evidence-based guidelines for preventing healthcareassosciated infections in NHS hospitals in England. J Hosp Infect 65:S1-S64

10. Marschall J, Mermel LA, Classen D, Arias KM, Podgorny K, Anderson DJ, Burstin H, Calfee DP, Coffin SE, Dubberke ER, Fraser V, Gerding DN, Griffin FA, Gross P, Kaye KS, Klompas M, Lo E, Nicolle L, Pegues DA, Perl TM, Saint S, Salgado CD, Weinstein RA, Wise R, Yokoe DS (2008) Strategies to prevent central line-associated bloodstream infections in acute care hospitals. Infect Control Hosp Epidemiol 29 Suppl 1:S22-30. doi:10.1086/591059

11. O'Grady NP, Alexander M, Burns LA, Dellinger EP, Garland J, Heard SO, Lipsett PA, Masur H, Mermel LA, Pearson ML, Raad, II, Randolph AG, Rupp ME, Saint S (2011) Guidelines for the Prevention of Intravascular Catheter-related Infections. Clin Infect Dis 52 (9):e162-e193. doi:cir257 [pii]

$10.1093 / \mathrm{cid} / \mathrm{cir} 257$

12. Traore O, Liotier J, Souweine B (2005) Prospective study of arterial and central venous catheter colonization and of arterial- and central venous catheter-related bacteremia in intensive care units. Critical Care Medicine 33 (6):1276-1280

13. Lucet JC, Bouadma L, Zahar JR, Schwebel C, Geffroy A, Pease S, Herault MC, Haouache H, Adrie C, Thuong M, Francais A, Garrouste-Orgeas M, Timsit JF (2010) Infectious risk associated with arterial catheters compared with central venous catheters. Critical Care Medicine 38 (4):1030-1035 
1 14. Gowardman JR, Lipman J, Rickard CM (2010) Assessment of peripheral arterial

2 catheters as a source of sepsis in the critically ill: a narrative review. J Hosp Infect 75 (1):12-

3 18. doi:10.1016/j.jhin.2010.01.005

4 15. Maki DG, Weise CE, Sarafin HW (1977) A semiquantitative culture method for

5 identifying intravenous catheter-related infections. New England Journal of Medicine $6 \quad 296: 1305-1309$

7 16. Sogin ML, Huse SM, Huber JA, Morrison HG, Mark Welch D (2007) Accuracy and 8 quality of massively parallel DNA pyrosequencing. Genome Biology 8 (7). doi:10.1186/gb9 2007-8-7-r143

10 17. Rafii F, Franklin W, Cerniglia CE (1990) Azoreductase activity of anaerobic bacteria isolated from human intestinal microflora. Appl Environ Microbiol 56 (7):2146-2151

18. Aziz RK, Bartels D, Best AA, DeJongh M, Disz T, Edwards RA, Formsma K, Gerdes S, Glass EM, Kubal M, Meyer F, Olsen GJ, Olson R, Osterman AL, Overbeek RA, McNeil LK, Paarmann D, Paczian T, Parrello B, Pusch GD, Reich C, Stevens R, Vassieva O, Vonstein V, Wilke A, Zagnitko O (2008) The RAST Server: rapid annotations using subsystems technology. Bmc Genomics 9:75. doi:10.1186/1471-2164-9-75

19. Fridkin SK, Welbel SF, Weinstein RA (1997) Magnitude and prevention of nosocomial infections in the intensive care unit. Infectious disease clinics of North America 11 (2):479496

20. Kaplan JB, Meyenhofer MF, Fine DH (2003) Biofilm growth and detachment of Actinobacillus actinomycetemcomitans. J Bacteriol 185 (4):1399-1404

21. Musken M, Di Fiore S, Romling U, Haussler S (2010) A 96-well-plate-based optical method for the quantitative and qualitative evaluation of Pseudomonas aeruginosa biofilm formation and its application to susceptibility testing. Nat Protoc 5 (8):1460-1469. doi:10.1038/nprot.2010.110

22. Sabat G, Rose P, Hickey WJ, Harkin JM (2000) Selective and sensitive method for PCR amplification of Escherichia coli 16S rRNA genes in soil. Appl Environ Microbiol 66 (2):844-849. doi:Doi 10.1128/Aem.66.2.844-849.2000

23. Zhu B, Liu H, Tian WX, Fan XY, Li B, Zhou XP, Jin GL, Xie GL (2012) Genome Sequence of Stenotrophomonas maltophilia RR-10, Isolated as an Endophyte from Rice Root. Journal of Bacteriology 194 (5):1280-1281. doi:Doi 10.1128/Jb.06702-11

24. Crossman LC, Gould VC, Dow JM, Vernikos GS, Okazaki A, Sebaihia M, Saunders D, Arrowsmith C, Carver T, Peters N, Adlem E, Kerhornou A, Lord A, Murphy L, Seeger K, Squares R, Rutter S, Quail MA, Rajandream MA, Harris D, Churcher C, Bentley SD, Parkhill J, Thomson NR, Avison MB (2008) The complete genome, comparative and functional analysis of Stenotrophomonas maltophilia reveals an organism heavily shielded by drug resistance determinants. Genome Biol 9 (4):R74. doi:10.1186/gb-2008-9-4-r74

25. Colvin KM, Gordon VD, Murakami K, Borlee BR, Wozniak DJ, Wong GCL, Parsek MR (2011) The Pel Polysaccharide Can Serve a Structural and Protective Role in the Biofilm Matrix of Pseudomonas aeruginosa. Plos Pathogens 7 (1). doi:ARTN e1001264

DOI 10.1371/journal.ppat.1001264

26. Vasseur P, Vallet-Gely I, Soscia C, Genin S, Filloux A (2005) The pel genes of the Pseudomonas aeruginosa PAK strain are involved at early and late stages of biofilm formation. Microbiology-Sgm 151:985-997. doi:DOI 10.1099/mic.0.27410-0

27. Piddock LJV (2006) Multidrug-resistance efflux pumps - not just for resistance. Nature Reviews Microbiology 4 (8):629-636. doi:Doi 10.1038/Nrmicro1464

28. Maroye P, Doermann HP, Rogues AM, Gachie JP, Megraud F (2000) Investigation of an outbreak of Ralstonia pickettii in a paediatric hospital by RAPD. J Hosp Infect 44 (4):267272 
1 29. Sundaram S, Lewis M, Eisenhuth J, Howard G, Larson B (2002) Method for qualifying 2 microbial removal performance of 0.1 micron rated filters - Part IV: Retention of 3 Hydrogenophaga pseudoflava (ATCC 700892) and Ralstonia pickettii (ATCC 700591) by

$4 \quad 0.2$ and $0.22 \mathrm{mu}$ m rated filters. Pda Journal of Pharmaceutical Science and Technology 56 5 (3):150-171

6 30. Ryan MP, Pembroke JT, Adley CC (2006) Ralstonia pickettii: a persistent gram-negative 7 nosocomial infectious organism. J Hosp Infect 62 (3):278-284. doi:S0195-6701(05)00371-3 $8 \quad$ [pii] $10.1016 /$ j.jhin.2005.08.015

9 31. Labarca JA, Trick WE, Peterson CL, Carson LA, Holt SC, Arduino MJ, Meylan M, 10 Mascola L, Jarvis WR (1999) A multistate nosocomial outbreak of Ralstonia pickettii 11 colonization associated with an intrinsically contaminated respiratory care solution. Clin 12 Infect Dis 29 (5):1281-1286

13 32. Fernandez C, Wilhelmi I, Andradas E, Gaspar C, Gomez J, Romero J, Mariano JA, Corral 14 O, Rubio M, Elviro J, Fereres J (1996) Nosocomial outbreak of Burkholderia pickettii 15 infection due to a manufactured intravenous product used in three hospitals. Clin Infect Dis $1622(6): 1092-1095$

17 33. Stelzmueller I, Biebl M, Wiesmayr S, Eller M, Hoeller E, Fille M, Weiss G, Lass-Floerl 18 C, Bonatti H (2006) Ralstonia pickettii - innocent bystander or a potential threat? Clinical 19 Microbiology and Infection 12 (2):99-101. doi:10.1111/j.1469-0691.2005.01309.x

20 34. Moreira BM, Pellegrino FLPC, Schirmer M, Velasco E, de Faria LM, Santos KRN (2008) 21 Ralstonia pickettii bloodstream infections at a Brazilian cancer institution. Current 22 Microbiology 56 (3):219-223. doi:10.1007/s00284-007-9060-1 